\title{
EDITORIAL
}

\section{¿POR QUÉ ES NECESARIA UNA REFORMA DE LA EDUCACIÓN SUPERIOR CHILENA?}

\author{
Dr. Emilio Rodríguez-Ponce ${ }^{1}$
}

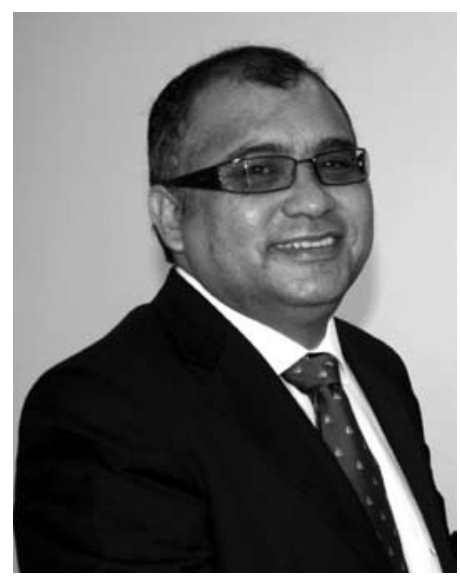

Dr. Emilio Rodríguez-Ponce

Treinta años atrás se llevó a cabo una reforma de la educación superior en Chile la que consistentemente con el accionar del gobierno de aquella época, no dio lugar a discusión ni debate democrático de ningún tipo. Dicha reforma, simplemente, se diseñó y se impuso. Los posteriores gobiernos democráticos, que emergen a partir de 1990, paradójicamente avanzan, sin solución de continuidad, en la misma lógica impulsada por el Gobierno Militar. Ciertamente, el poder ejecutivo desde el retorno a la democracia sólo ha realizado ajustes menores al modelo de los años 80. Tales ajustes, lejos de debilitar el sistema y diseño previos, lograron su consolidación en el mundo de las ideas, al menos entre los miembros del "stablishment", y sobre todo en el campo de los hechos reales.

La filosofía de aquella reforma tuvo sus bases conceptuales y su sustento en el principio de libertad para elegir, y en la lógica de mercado como mecanismo de coordinación y asignación de recursos. En efecto, tal como lo plantea Lavados Montes (2005), el modelo de educación superior chilena, implementado a partir de 1981, emerge desde una perspectiva que considera a la educación y al conocimiento como bienes que pueden ser transados en el mercado. Así, el pago de aranceles por docencia de pregrado, postgrado y por prestaciones de servicios son expresiones concretas de este enfoque. El mercado, en la filosofía subyacente al modelo, se entiende como el principal coordinador y responsable del derrotero que ha de tomar la educación superior, ya que a través de la competencia y del mecanismo de precios se habrá de regular la calidad y la pertinencia del sistema y de sus integrantes.

Bajo esta lógica se emprendieron una serie de amplias reformas estructurales. Fueron reformas de la educación superior, en el sentido más profundo y auténtico de la palabra, ya que se modificaron radicalmente las estructuras, los contenidos y los fines de las instituciones de educación superior.

Así como lo plantea Capel (2005), la educación superior se segmentó en universidades, institutos profesionales y centros de formación profesional. De este modo, las ocho instituciones tradicionales vieron el nacimiento de varias decenas de universidades derivadas y privadas que, sumadas a los institutos profesionales y centros de formación técnica, elevaron la oferta académica a niveles inéditos e insospechados.

Por este camino, en el cual el mercado es el coordinador de la educación superior, el aporte del Estado al sistema de educación terciaria llegó sólo al 0,3\% del producto interno bruto en el año 2006, equivalente al $14 \%$ del gasto total en el

Rector, Universidad de Tarapacá, Investigador Principal - Proyecto FONDECYT 1090116. 
área. En otras palabras, en ese año las familias han debido financiar el $86 \%$ del costo de la educación superior del país. En todo caso, el principal logro que exhibe el sistema actual es que, a pesar de la menor inversión del Estado en educación terciaria, la cobertura de la población entre 18 y 24 años ha llegado a niveles históricos del 50\%, destacándose que 7 de cada 10 estudiantes que están en educación superior son los primeros de sus familias en alcanzar este nivel de estudios (Lavín, 2011).

Condicionados a los supuestos del modelo impuesto en Chile pareciera justo, desde el punto de vista social, que las personas que acceden a la educación superior deban pagar por sus estudios, ya que a fin de cuentas sus ingresos permanentes serán entre 3 a 5 veces mayores que los de sus contemporáneos que no logren un título universitario. Por cierto, las instituciones de educación superior ofrecen oportunidades de estudiar y obtener una certificación valorada en el mercado del trabajo y que conducirá a mejores empleos y a movilidad social. La educación superior ha sido y es todavía una gran plataforma para la movilidad social en Chile (RodríguezPonce, 2009 a).

En consecuencia, pareciera ser que quien obtiene una educación superior tiene una responsabilidad ineludible para con la sociedad y esa responsabilidad implica retornar solidariamente lo que se ha invertido en esa persona.

En este contexto, bajo una mirada economicista, pareciera posible plantear que el sistema chileno ha funcionado exitosamente, puesto que invirtiéndose, prácticamente, el equivalente a $1 / 4$ de la proporción del producto interno bruto que se destinaba en 1973 a la educación superior, la cobertura se ha multiplicado por 3 , con lo cual: cada peso del Estado en educación terciaria es, ahora, 12 veces más productivo que en ese tiempo. Más aún, se podría argumentar, con cierta razón, que la calidad de las instituciones no ha sido descuidada. Considérese, por ejemplo, que las universidades privadas han pasado procesos largos de evaluación de su calidad; primero, logrando su licenciamiento por parte del Consejo Superior de Educación, y luego validando dicho licenciamiento mediante su respectiva acreditación.

Por lo tanto, el sistema ha mejorado en cobertura, y las instituciones universitarias privadas parecieran haber demostrado calidad ante las instancias evaluadoras como el Consejo Superior de Educación y la Comisión Nacional de Acreditación. Incluso, una mirada objetiva de las universidades derivadas muestra que, prácticamente en cada Región del país, éstas han consolidado sus cuadros académicos en niveles superiores a los de la década de los 80 y su equipamiento e infraestructura es mayor en cantidad y calidad respecto de esa época.

¿Qué es lo que está mal en este modelo que exhibe logros que parecen ser tan evidentes? ¿Por qué centenares de miles de personas han salido a las calles a pedir una mejor educación y una educación esencialmente pública? ¿Se trata simplemente de personas egoístas y poco solidarias que mediante la presión social quieren evitar pagar por una educación que les proporcionará, sin duda, mayores ingresos en el futuro? ¿Se trata sólo de grupos anárquicos que ven una oportunidad para castigar al sistema?

Aquí se intentarán esbozar algunas respuestas o, al menos, algunas reflexiones que pueden dar luces sobre las interrogantes planteadas. Ciertamente, pueden brindarse múltiples prismas para generar respuestas a estas interrogantes. Sin embargo, en estas reflexiones se configuran a partir de la mirada de la dirección estratégica.

En consecuencia, siguiendo la lógica de Grant (1996), que sugiere que desde la perspectiva de la dirección estratégica es fundamental disponer de objetivos claros y sencillos, una de las primeras cuestiones a considerar es si los objetivos estratégicos pregonados públicamente por los ideólogos del modelo se cumplen o no. La cuestión a dilucidar es: si efectivamente existe o no libertad para elegir, si el modelo genera bienes valiosos para los agentes y si, finalmente, existen niveles satisfactorios de cobertura, calidad y equidad en el modelo chileno de educación superior.

Para este efecto, se realizarán una serie de preguntas estratégicas a las que se intentará dar respuesta, considerando los determinantes estructurales y los efectos y alcances de los elementos que subyacen en dichas respuestas.

La primera pregunta es: ¿los estudiantes de la educación superior chilena tienen la libertad para elegir en dónde realizar sus estudios terciarios? Esta interrogante alude al corazón del sistema chileno, ya que la libertad para elegir implica dotar a cada potencial estudiante de la capacidad y del poder para obrar según su propia voluntad, haciéndose responsable de su elección. 
La verdad es que en la educación superior chilena no hay libertad de elección salvo para los miembros de los grupos altamente privilegiados desde la perspectiva social y económica. En efecto, la educación en enseñanza básica y media recibida es el principal determinante de los resultados de los procesos de selección universitaria; asimismo, la capacidad de pago de los aranceles de matrícula y la posibilidad de manutención por los años de estudio incidirán en la elección final de los aspirantes a educación terciaria. Además, considérese que la propia calidad de la formación recibida en enseñanza básica y media está determinada por la condición social y económica de las personas.

Luego, con las excepciones normales en el campo de las ciencias sociales, la condición socioeconómica determina de manera significativa la calidad de la enseñanza básica y media, la que a su vez condiciona los resultados de los procesos de selección universitaria. Por ejemplo, los colegios Top-60 en la prueba de selección universitaria rendida el año 2010, son: en el $95 \%$ de los casos colegios particulares pagados; en el 3,4\% son colegios municipalizados, y el 1,6\% de los casos corresponde a un colegio particular subvencionado (DEMRE, 2011).

Ahora bien, la misma situación social y económica pone una traba adicional al merito académico. En efecto, aunque los estudiantes logren derrotar sus condiciones de entrada, deben disponer de los recursos adicionales a los costos de matrícula para garantizar su manutención personal durante los años que duren sus estudios universitarios, cuestión que restringe sus opciones reales de elección.

Por lo tanto, no cabe duda que las posibilidades de acceso y la calidad de la educación disponible para los jóvenes de Chile se asocian indisolublemente a la condición socioeconómica de su núcleo familiar. Por cierto, dos jóvenes de igual talento académico y calidad intelectual no tienen las mismas oportunidades de elección si sus condiciones económicas son significativamente distintas. El joven con peor condición social y económica tendrá, con las excepciones que confirman la regla, una educación de menor calidad en la enseñanza básica, media y universitaria. Consecuentemente, su trabajo será de menor calidad y recibirá menores ingresos que los jóvenes de igual talento, pero de una condición social y económica significativamente superior. Habrá movilidad social, ya que el joven de menores recursos: "saldrá adelante en la vida", pero sus oportunidades no se acercarán, siquiera en lo esencial, a las opciones de otras personas de igual talento, cuya diferencia fundamental son las condiciones de nacimiento. Benavente et al. (2004) muestran que en un conjunto de carreras universitarias hay diferencias de remuneraciones significativas dependiendo de la universidad en la cual se obtuvo el título correspondiente.

La situación es tanto o más grave cuando se incorpora el componente regional al análisis. Un estudiante talentoso de los quintiles más desposeídos de Arica o Punta Arenas no tiene las mismas oportunidades de elección que un estudiante del mismo talento y de las mismas condiciones, que vive en la ciudad de Santiago. Por ejemplo, entre los colegios Top-60 de la última prueba de selección universitaria no existe ni siquiera un colegio de ninguna de las regiones de Chile que sea municipalizado ni particular subvencionado (DEMRE, 2011).

Así, es fácil demonizar al mercado como el responsable de los males de la educación superior en Chile, pero el culpable no es el mercado. Al mercado no se le puede pedir imposibles. Efectivamente, si como se ha dicho en la ideas anteriormente expuestas, la condición socioeconómica determina las posibilidades de elección real; si los costos de la educación no se pueden estandarizar ya que el capital social, económico y cultural de la población estudiantil es un determinante estructural de sus posibilidades de aprendizaje; si no existe una perfecta movilidad de recursos en el sistema de educación superior; si existen costos elevados de traslado para los usuarios del sistema; y si la asimetría de información que existe en el sistema no permite a los estudiantes y a sus familias adoptar, necesariamente, las mejores decisiones, entonces no se le puede pedir al mercado que obre milagros.

Luego, el modelo actual no cumple con su objetivo primario de dar a las personas libertad para elegir. En efecto, en este sistema de educación superior las personas eligen lo que pueden y lo que pueden está condicionado, antes que todo, por la cuna en la cual nacieron.

Si bien la reflexión anterior parece invalidar, en sí misma, la pertinencia estratégica del modelo actual, en cualquier caso es importante avanzar en la segunda interrogante asociada a los objetivos del sistema de educación superior: ¿el modelo 
genera bienes valiosos para los individuos y para la sociedad? Esta pregunta alude a otra cuestión fundamental en tanto la existencia de bienes privados, es decir, valiosos para los individuos se puede asociar a una lógica de coordinación mediante el mercado y el mecanismo de precio; sin embargo, la potencial existencia de bienes públicos generados por el modelo rompe con la pertinencia de una lógica estricta de mercado y da lugar a la necesaria existencia de un rol determinante y activo del Estado.

En la sociedad del conocimiento tanto capital como trabajo son requeridos para la producción de bienes, pero el conocimiento es la base o esencia de la ventaja competitiva en la nueva economía. Por lo tanto, desde esta perspectiva, la educación superior debe asumir un conjunto de desafíos centrales. Primero, las instituciones de educación terciaria deben constituirse en un elemento básico para generar mayores niveles de competitividad en el país. En efecto, en la sociedad del conocimiento y en un ambiente globalizado la formación de capital humano avanzado, así como la investigación, el desarrollo y la innovación constituyen pilares fundamentales de la ventaja competitiva para la nación, las organizaciones y las personas. Segundo, las instituciones de educación superior deben constituirse en una fuente esencial de oportunidades de formación continua y movilidad social. Ciertamente, la rentabilidad privada de la educación superior es aún significativa. Tercero, las instituciones de educación superior deben velar por la calidad y pertinencia de su oferta académica, cuestión de la mayor importancia para generar programas de formación e investigación que efectivamente impacten sobre la capacidad competitiva del país y permitan la inserción exitosa de jóvenes de familias vulnerables en el mercado del trabajo. Cuarto, las instituciones de educación superior deben vincularse con su medio aportando al desarrollo de la cultura, las artes, las letras y el desarrollo territorial (Rodríguez Ponce, 2009 b).

Es indudable que la educación superior chilena puede generar bienes privados, pero también genera bienes públicos. Por ejemplo, un año más de escolaridad tiene un impacto sobre la productividad individual que bordea entre 6 y 15\% (Dowrick, 2002; Bassanini y Scarpetta, 2002; Mankiw et al., 1992), cuestión que debería conducir a una mejor remuneración para los individuos, pero ese mismo año adicional de escolaridad implica un incremento del potencial del producto interno bruto en proporciones que, en el largo plazo, resultan altamente significativas contribuyendo de esta forma a un mayor bienestar y progreso para la sociedad en su conjunto.

Por su parte, la creación de conocimiento asociada a investigación, desarrollo e innovación permite la creación de nuevos productos y tecnologías y, por ende, tiene una alta rentabilidad privada y social. Por ejemplo, los trabajos de Nadiri (1993) y de Lichtenberg y Siegel (1991) muestran rentabilidades privadas superiores al $20 \%$ para las inversiones en investigación y desarrollo en diferentes sectores económicos. Por su parte, los trabajos de Frantzen (2000) y de Lichtenberg y Van Pottelsberghe (1996) muestran una rentabilidad social superior al $50 \%$ para las inversiones en investigación y desarrollo. Esto no solo ocurre en los países desarrollados, ya que Benavente (2006) demuestra que la situación se repite en una economía emergente como la de Chile. En consecuencia, la investigación, el desarrollo y la innovación son bienes que proporcionan beneficios privados, pero también bienes públicos.

Finalmente, la educación terciaria constituye una fuente de movilidad social con una rentabilidad privada aún atractiva para las personas, principalmente en los países emergentes (Duryea et al., 2001; Fleet, 2007), incluso en localidades extremas (Brunner et al., 2006). Por lo demás, la formación de capital humano avanzado permite la consolidación territorial de las distintas regiones de un país. Como lo plantea Bloom et al. (2006), la educación superior tiene una serie de beneficios para una sociedad tanto desde la perspectiva económica como desde la perspectiva social. Los beneficios económicos se asocian a: mayor recaudación de impuestos; mayor potencial de productividad para el país; mayores niveles de demanda agregada; mayores niveles de ahorro; mayor flexibilidad de la fuerza de trabajo, entre otros similares. Por su parte, los beneficios sociales se asocian a: menores tasas de criminalidad; mejor calidad de vida cívica; mayor cohesión social; aprecio por la diversidad; mejores condiciones de salud y expectativas de vida, entre otros.

En este punto de la reflexión emergen, con fuerza, tres consideraciones básicas. Primero, existe la tendencia a considerar a la educación superior sólo como docencia, despreciando la relevancia y el rol esencial que las instituciones terciarias tienen 
en la investigación, el desarrollo y la innovación. Asimismo, no se suele dimensionar el verdadero valor del rol de la vinculación con el medio de las instituciones de educación superior. Probablemente, el hecho que muy pocas instituciones chilenas cumplan estos roles con solvencia, hace que se tienda a menoscabar conceptualmente y en los hechos concretos los roles de formación de postgrado, investigación y vinculación con el medio. Segundo, la educación superior es un bien cuasipúblico, que tiene componentes de bien privado y eso no puede ni debe ser negado; pero tampoco puede ni debe ser negado su impacto como bien público, ya que incluso desde la perspectiva más reduccionista de la docencia de pregrado y de los impactos económicos se pueden comprender los impactos sobre la productividad del país, la recaudación fiscal, y los mayores niveles de ahorro que genera la educación terciaria. Ahora bien, la educación superior tiene un rol vital como bien público, mucho mayor todavía cuando se incorpora la visión más integradora de los roles de investigación, desarrollo e innovación. Tercero, el conocimiento tiene valor en sí mismo, porque en el conocimiento está la fuente del progreso no sólo económico, sino que las bases de la cultura y del desarrollo de la sociedad. El afán y la vocación por aprender e investigar es el sustento del desarrollo de la humanidad; ciertamente, muchos de los principales descubrimientos, inventos y reflexiones no se llevaron a cabo bajo la lógica de la búsqueda de la productividad, sino que emergieron del ingenio y la curiosidad de personas con mentes entrenadas para crear y proyectar su saber.

La tercera pregunta desde la mirada estratégica es: ¿existen niveles satisfactorios de cobertura, calidad y equidad en el modelo chileno de educación superior? Esta pregunta se vincula con una revisión más profunda de los resultados más difundidos del modelo.

Respecto de la cobertura, se sabe que ésta alcanza a más de un millón de estudiantes, lo que representa cerca del $50 \%$ de la población entre 18 y 24 años. Si bien es cierto que en 20 años se quintuplicó la cobertura en el $30 \%$ de las personas con menores ingresos, lo cual es sin duda una buena noticia, no es menos cierto que, en términos concretos, la proporción esperada de acceso a la educación superior para un estudiante del $10 \%$ más vulnerable de la población es sólo un $19,1 \%$, mientras que un estudiante del decil más rico ingresa en una proporción del 93,3\% (Lavín, 2011).

Los más desposeídos no sólo ingresan en menor proporción a la educación superior, sino que la posibilidad de ingresar a las universidades es mayor para las personas de mejor situación socioeconómica. Junto a esta cobertura que muestra progreso y, a la vez, falta de equidad, es menester considerar que la deserción en la educación superior chilena es del 52\% (Ugarte, 2011).

Esto implica que más de la mitad de los estudiantes que ingresan a estudiar educación terciaria nunca se gradúan. La duración real de las carreras suele ser 1,5 veces la duración teórica o ideal. Estas cifras dejan de manifiesto que el sistema fracasa en su cometido, ya que si bien tiene las puertas abiertas para el ingreso, las puertas de la salida al éxito están llenas de obstáculos. Por eso, cuando se dice que 7 de cada 10 personas que ingresan a la educación superior son primera generación familiar en lograrlo, resulta fundamental conocer la respuesta a cuántas de esas 7 personas finalmente se gradúan y en cuánto tiempo lo logran.

Probablemente, para algunas personas sea mejor no ingresar a la educación superior, que salir de ésta sin éxito, con los sueños y las ilusiones rotas, y con un endeudamiento que acechará y limitará su consolidación familiar y económica.

El sistema chileno muestra una cobertura creciente y en progreso, pero ésta es una falsa cobertura si se entiende que más de la mitad de las personas que están en educación superior no se graduarán nunca. En cuanto a equidad el sistema es claramente deficiente.

Respecto de la calidad, todas las universidades privadas pasaron por largos procesos de evaluación por parte del Consejo Superior de Educación. Sin embargo, este hecho no ha sido garantía de calidad. Por cierto, instituciones licenciadas no fueron capaces de pasar procesos de acreditación. La misma acreditación que en algún momento mostró méritos innegables en cuanto a su impacto en los procesos de mejoramiento en las instituciones (IPSOS, 2010), cada vez ha perdido mayor vigor y credibilidad.

Por ejemplo, en los procesos de acreditación institucional de fines de 2010 todas las instituciones que postularon fueron acreditadas. Lo anterior podría implicar que ha existido un mejoramiento notable en las instituciones de educación terciaria 
en Chile o que, simplemente, la vara de medida y los niveles de exigencia han caído a niveles lamentables. Ahora bien, cuando se observa el estado y el bajo nivel de calidad con el cual operan sedes de instituciones llamadas universitarias, sobre todo en regiones, se puede reconocer que lo que ha ocurrido en realidad es que el sistema de aseguramiento de la calidad en Chile no está garantizando el cumplimiento de estándares mínimos, al menos en las sedes.

Sin embargo, esto no sólo ocurre en las acreditaciones institucionales. Recientemente, a fines del año 2010, una institución universitaria acreditó su carrera de Medicina ante una agencia privada, por dos años. Esto significaría que en dicha carrera hay garantía de calidad. Sin embargo, el $48 \%$ de sus titulados, luego de 7 largos años de estudio, reprobó el examen médico nacional, dejando en evidencia su escaso nivel de preparación y conocimiento (Rodríguez-Ponce, 2011). Una reflexión adicional a este marco de referencia y análisis es saber ¿a quiénes atenderán estos médicos deficientemente formados? Probablemente a quienes no puedan costear los servicios de médicos de mayor jerarquía académica e intelectual.

La relación cliente-proveedor entre instituciones de educación superior y agencias privadas, de acreditación de carreras y programas, genera una relación intrínsecamente compleja, con la existencia de riesgos implícitos similares a los que la evidencia empírica muestra en el caso de las instituciones privadas clasificadoras de riesgo, cuya responsabilidad en la reciente crisis económica mundial ha sido altamente significativa (Instituto Iberoamericano de Mercado de Valores, 2009).

En consecuencia, el sistema de educación superior chileno muestra una cobertura creciente, pero esconde la realidad de altos niveles de deserción y una prolongada permanencia o duración excesiva de los estudios. Por lo demás, no hay equidad en el acceso, y la calidad institucional, de las carreras y de los programas, no está garantizada en un sistema de aseguramiento de la calidad que pierde consistencia y credibilidad.

Se hace así imprescindible, sin lugar para las ambigüedades, una profunda reforma a la educación superior chilena. Dicha reforma debe tener como elemento central la definición de objetivos estratégicos éticos y coherentes con una sociedad democrática. En efecto, los objetivos estratégicos de un sistema de educación superior debe asociarse, al menos, a las siguientes dimensiones: provisión de bienes para el país; equidad en todos los niveles y ámbitos del sistema, y aseguramiento de la calidad.

Respecto de la provisión de bienes, lo más importante es romper con la ceguera conceptual de un cierto sector dominante del "stablishment", que no puede ni quiere ver más que los bienes privados que genera el sistema chileno. Un verdadero sistema de educación superior generará bienes privados, tales como mayor educación y potencial productivo para los individuos de una sociedad, lo que ampliará sus horizontes y oportunidades laborales, pero la educación superior generará, en primer lugar, bienes públicos. Un objetivo estratégico para un sistema educacional, prácticamente en cualquier parte del mundo, es: contribuir al mejoramiento de la sociedad; mejorar la calidad de vida cívica; fortalecer los principios democráticos y del humanismo; lograr mayor cohesión social, etc. Estos objetivos no están reñidos con los beneficios privados que proporciona la educación superior ni con otros bienes públicos de carácter económico, tales como: los impactos sobre la productividad del país; la recaudación fiscal, y los mayores niveles de ahorro que genera la educación terciaria, entre otros similares.

Un objetivo de este tipo supone que el Estado tiene la obligación moral y el imperativo estratégico de financiar la educación de pregrado, postgrado, la investigación, y la vinculación con el medio. Por lo tanto, la gratuidad de la educación de pregrado para los alumnos talentos de los grupos social y económicamente vulnerables es esencial tanto en el pregrado como en el postgrado. Pero no basta con esto, el Estado tiene un rol fundamental en la investigación y en la vinculación de la educación terciaria con su medio.

Para estos propósitos resulta vital disponer de Universidades del Estado y Centros de Formación Técnica del Estado en cada Región del país, los cuales deben constituirse en instituciones que ofrezcan, en lo pertinente a su misión, formación de pregrado, postgrado, investigación y vinculación con el medio gratuita para las personas de los grupos vulnerables y en niveles de calidad, equivalentes al máximo nacional. Todo Chile es Chile, no sólo Santiago. Todos los chilenos merecen un sistema de educación superior que cumpla con su rol.

Por su parte, la equidad tiene al menos las dimensiones: social y económica y la regional. Un 
sistema de educación superior debería garantizar el acceso de las personas talentosas, con independencia del lugar en el que nacieron, lugar en sentido de la condición social, o cuna económica de las personas, y lugar en sentido de la región del país. Por lo tanto, el sistema debe dejar de lado los reduccionismos actuales, y las reflexiones convenientes para los intereses privados que sostiene el grupo de mayor poder comunicacional y económico en el "stablishment". Las personas tienen talentos equivalentes en cada Región del país, tanto quienes estudian en colegios privados como aquellos que lo hacen en escuelas públicas. El talento, al revés de lo que hace el sistema de educación superior chileno, no discrimina por sexo, por nivel social, ni por territorio.

Por lo tanto, se requiere que en cada Región del país existan Universidades del Estado y Centro de Formación Técnica del Estado, del mayor nivel de calidad, con la suficiente amplitud de áreas y complejidad para realizar todas las labores auténticamente públicas, en lo pertinente a sus respectivas misiones institucionales, tales como: docencia de pregrado, postgrado, investigación,y vinculación con el medio. Además, se requiere que estas instituciones asuman el compromiso de un acceso garantizado a los mejores alumnos de sus respectivos cursos, dejando de lado la prueba de selección universitaria como único criterio de selección. Sin embargo, no basta con esto, adicionalmente, se requiere que el sistema de educación superior, en subsidio al sistema de enseñanza básica y media, genere programas remediales que aseguren niveles de éxito y titulación superiores al 70\%, como ocurre en los países desarrollados.

Finalmente, el sistema debería dar garantía pública de la calidad de las instituciones. Ciertamente, las entidades del Estado deberían tener, por definición, los más altos estándares de calidad, amplitud y complejidad. Estas instituciones deberían rendir cuenta pública de su quehacer y evaluar su calidad sistemáticamente con la meta de constituirse en referentes de calidad nacional y en Iberoamérica. Pero también es posible que operen en el sistema otras instituciones de tipo privado, las cuales deberían rendir pruebas permanentes, sistemáticas e ininterrumpidas de su calidad, no sólo a nivel de la consistencia interna de su propósitos, fines y accionar, sino que a nivel del cumplimiento de estándares objetivos y cuantificables de calidad. No sólo a nivel de sus procesos, sino que de sus resultados.

\section{Literatura Citada}

Bassanini, A. y Scarpetta, S.

2002 "Does human capital matter for growth in OECD countries? A pooled mean-group approach". Economics Letters 74, pp. 399-405.

Benavente, J.; Rappoport, D. y Meller, P.

2004 "Rankings de universidades chilenas según los ingresos de sus titulados". Banco Central de Chile, Documentos de Trabajo, No 306, diciembre, 2004.

Benavente, J.

2006 "The Role of research and innovation in promoting productivity in Chile". Economics of Innovation and New Technology, v. 15, No 4-5, pp. 301-315.

Bloom, D.; Canning, D. y Chan, K.

2006 "Higher education and economic development in Africa". Harvard University.

Brunner JJ.; Bonnefoy, J.; Elacqua G. y González, S. 2006 “Capital Humano en la Región de Tarapacá”, Universidad Adolfo Ibáñez, 75 pp.

Capel, A.

2005 "Cambios en el sistema de educación superior en Chile y sus efectos en la gestión financiera". Revista de Calidad en la Educación. Consejo Superior de Educación N ${ }^{\mathbf{o}} 22$, pp. 283-329.
DEMRE

2011 "Proceso de admisión 2011, resultados de selección". Universidad de Chile, Vicerrectoría de Asuntos Académicos, Departamento de Evaluación, Medición y Registro Educacional. Santiago, 12 de enero de 2011.

Dowrick, $\mathrm{S}$.

2002 "Ideas and education: Level or Growth Effects? Thirteenth Annual East Asian Seminar on Economics Productivity, Melbourne, June 20-22.

Duryea, S. y Pagés, C.

2001 "Human Capital Policies: What they Can and Cannot do for Productivity and Poverty-reduction in Latin America". Forthcoming in: American Foreign Economic Relations: Policy Dilemmas and Opportunities. Miami, United States: North South Press.

Fleet, N.

2007 "Rentabilidad privada de la educación superior". Working Paper. Comisión Nacional de Acreditación. Santiago de Chile. V. 5, pp. 29.

Frantzen, D.

2000 "Innovation, international technological diffusion and changing influence of R\&D on productivity". Cambridge Journal of Economics, v. 24, 193-210. 
Grant, R.

1996 "Toward a knowledge-based theory of the form". Strategic Management Journal, v. 17, Winter special issue, pp. 109-122.

IIMV

2009 "Rol de las Clasificadoras de Riesgo en la Crisis Financiera Internacional". Instituto Iberoamericano del Mercado de Valores Santiago de Chile, 30 de octubre de 2009.

IPSOS

2010 "Informe final: Estudio exploratorio sobre efectos de la acreditación institucional en la calidad de la educación superior en Chile". Preparado para la Comisión Nacional de Acreditación, Santiago, abril, 2010.

Lavados Montes, J.

2005 "Desafíos pendientes de las políticas públicas en educación superior". Revista Calidad en la Educación. V. 22 $\mathrm{N}^{\mathrm{o}} 1$, pp. 23-36.

Lavín, J.

2011 "El modelo de educación superior para el Chile del futuro”. Presentación del Ministro de Educación en el salón de honor Congreso Nacional, 13 de enero, 2011.

Lichtenberg, F. y Siegel, D.

1991 "The impact of R\&D investment on productivity- New evidenve using R\&D - LRD Data", Economic Inquiry, 29, pp. 203-228.
Lichtenberg, F. y Van Pottelsberghe, B.

1996 "International R\&D Spillovers: A Re-Examination". NBER Working paper No 5668. Issued in July 1996.

Mankiw, N. Romer, D. y Weil, D.

1992 "Contribution to the empirics of economic growth". Quarterly Journal of Economics, v. 107, n. 2, pp. 407-437.

Nadiri, M.

1993. Innovations and Technological Spillovers, NBER Working Paper $\mathrm{N}^{\mathrm{o}} \mathrm{W} 4423$.

Rodríguez-Ponce, E.

2009 a "The role of universities in the knowledge society and globalization: Evidence from Chile". Interciencia, v. 34, n. 11, pp. 822-829.

Rodríguez-Ponce, E.

2009 b "Las universidades en la sociedad del conocimiento". pp. 35-68. En Arata Andreani, A. y Rodríguez Ponce, E. (2009) (Editores). Desafíos y perspectivas de la dirección estratégica de las instituciones universitarias. Ediciones CNA Chile. 549 pp.

Rodríguez-Ponce, E.

2011 "Sistema de acreditación y calidad de la educación superior". Presentación efectuada en Sala de Lectura, de la Cámara de Diputados, Congreso Nacional, Santiago. Lunes 23 de mayo, 2011.

Ugarte, J.

2011 "Desafíos de la educación superior chilena". http:// www.cedus.cl/?q=node/1634 